1 THE USE OF PLATELET-RICH PLASMA (PRP) TO TREAT CHRONIC TENDINOPATHIES: A TECHNICAL ANALYSIS

${ }^{1}$ JF Kaux, ${ }^{1} \mathrm{~T}$ Emonds-Alt, ${ }^{2} \mathrm{~S}$ Sport. ${ }^{1}$ Department of Physical Medicine and Sports Traumatology; ${ }^{2}$ FIFA Medical Centre of Excellence, University and University Hospital of Liège, Belgium

\subsection{6/bjsports-2018-ISSSMC2018.1}

Platelet-rich plasma (PRP) is blood plasma with a high concentration of autologous platelets which constitute an immense reservoir of growth factors. The clinical use of PRP is widespread in various medical applications. Although highly popular with athletes, the use of PRP for the treatment of tendinopathies remains scientifically controversial, particularly due to the diversity of products that go by the name of 'PRP.' To optimize its use, it is important to look at the various stages of obtaining PRP. In this literature review, we take a closer look at eight parameters which may influence the quality of PRP:

1. anticoagulants used to preserve the best platelet function,

2. the speed of centrifugation used to extract the platelets,

3. the platelet concentrations obtained,

4. the impact of the concentration of red and white blood cells on PRP actions,

5. platelet activators encouraging platelet degranulation and, hence, the release of growth factors, and

6. the use or nonuse of local anesthetics when carrying out infiltration.

In addition to these parameters, it may be interesting to analyze other variables such as

7. the use of ultrasound guidance during the injection with a view to determining the influence they have on potential recovery.

In conclusion, there is a lack of standardization in PRP preparation technique for tendinopathies. However, it appears that the use of a platelet concentration lower than 5 times the baseline and avoidance of leukocytes should be preferred.

\section{MENTAL WELLBEING IN ELITE MALE UNDER 23 SOCCER PLAYERS: A TEAM CASE STUDY}

\begin{abstract}
${ }^{1}$ Andy Richardson, ${ }^{1}$ Tom Clifford, ${ }^{2,3}$ Will Abbott, ${ }^{4}$ Thomas E Brownlee, ${ }^{5}$ Liam D Harper, ${ }^{5}$ Robert I Naughton. 'Institute of Cellular Medicine, School of Biomedical Sciences, Newcastle UK; ${ }^{2}$ School of Sport and Service Management, Brighton University, UK; ${ }^{3}$ Brighton and Hove Albion F.C, American Express Elite Performance Centre, Lancing, UK; ${ }^{4}$ Research Institute for Sport and Exercise Sciences, Liverpool Johns University, Liverpool, UK; ${ }^{5}$ School of Human and Health Sciences, University of Huddersfield, UK
\end{abstract}

10.1136/bjsports-2018-ISSSMC2018.2

Recent research suggests a high prevalence of mental health disorders in professional soccer players, including symptoms of anxiety, depression, distress and adverse eating behaviours. Whilst a limited number of studies address this issue, the existing evidence is alarming and highlights a need for further investigations to develop a better understanding of the causes and consequences of mental health problems in professional soccer players. Thus, the aim of this study was to examine the influence of physical and emotional stressors on mental wellbeing $(\mathrm{MW})$ in professional male soccer players. Using a longitudinal design, twenty-five male soccer players from the under 23 squad playing in the Premier League 2 division in the UK completed the Warwick-Edinburgh Mental Well-being Scale (WEMWBS) each week of the 2017/2018 season (37 weeks in total). Independent predictor variables of MW were injury, match selection (in the match squad), weekly training load (sum of total duration, total distance and total sprint distance) and win rate. Mean MW scores were calculated across the season for each player with a squad mean of $48 \pm 3.94$, which is below the England population norm of 51.6. Mean MW was significantly lower when injured vs. not-injured (43.6 \pm 5.0 vs $49.9 \pm 3.5 ; \mathrm{p}=0.01)$, but was not affected by selection, training load or match result. Multivariate regression models showed time out with injury to have the strongest influence on MW $\left(\mathrm{r}^{2}=0.40, \mathrm{p}<0.01\right)$, with longer periods of injury linked to lower MW scores. Matchday selection was the only other statistically significant variable, with a positive correlation between MW and selection $\left(\mathrm{r}^{2}=0.23, \mathrm{p}=0.02\right)$. Whilst elucidating the mechanisms by which time out with injury may affect MW was beyond the scope of this study, the association between injury and reduced MW highlights a need for mental health and wellbeing monitoring in injured soccer players. This could facilitate interventions and support to ensure health and performance are not negatively affected.

\begin{tabular}{l}
\hline 3 \\
INFLUENCE OF INPUT DATA ERRORS ON THE INVERSE \\
DYNAMICS ANALYSIS OF HUMAN LOCOMOTION: TIME- \\
SERIES CHANGES IN LOWER EXTREMITY JOINT \\
MOMENT AND POWER DURING RUNNING
\end{tabular}

Heung-Youl Kim. Physical Education Center, Nanzan University, Japan

\subsection{6/bjsports-2018-ISSSMC2018.3}

The purpose of this study was to investigate the influence of inaccuracies between the center of pressure (CoP) recorded from a force platform and the body segment parameters (BSP) calculated from the different anthropometric models on resultant joint moment and power. Biomechanical data while running were obtained for one healthy male subject. Using inverse dynamics procedures, the lower extremity joint moment and power were computed for five successful trials. Thereafter, recalculations of the joint kinetic values were carried out under the following conditions: (1) five different BSP models (i.e., Ae (Japanese model), Vaughan, Chandler, Zatsiorsky, and De Leva), and (2) The CoP shifted in the anteriorposterior direction by $\pm 5 \%$ and $\pm 10 \%$ from the actual location. Changes of the joint kinetics resulting from difference in BSP models showed similar changes in all joints. There was similar changes between the curves, and statistically significant difference in the results was not observed. On the other hand, the anterior shifts in the CoP caused an elevation in the ankle plantal flextion and hip extension moment curves and a decrease in the knee extension moment curves. The posterior shifts in the CoP had the opposite effects. But the times of occurrence of the peak values were not affected. When CoP was shifted in the anterior direction, the ankle and hip joint power generations were increased, and decreased in the posterior direction. In the knee joint power generation had the opposite results. The adjustments in the CoP produced changes in the observed joint moment variables of $14 \%$ and $34 \%$, on average for the $\pm 5 \%$ and $\pm 10 \%$ shifts, respectively. On the other hand, in the joint powers, greater power generation was observed with hip joint than other joint powers. Using a one-way repeated measures ANOVA, when CoP 